\title{
Investigations of solar plasma in the interior and corona from Solar Dynamics Observatory
}

\author{
A. G. Kosovichev \\ W. W. Hansen Experimental Physics Laboratory, Stanford University, \\ Stanford, CA 94305, AKosovichev@solar.stanford.edu
}

\begin{abstract}
The Sun is a plasma laboratory for astrophysics, which allows us to investigate many important phenomena in turbulent magnetized plasma in detail. Solar Dynamics Observatory (SDO) launched in February 2010 provides unique information about plasma processes from the interior to the corona. The primary processes of magnetic field generation and formation of magnetic structures are hidden beneath the visible surface. Helioseismic diagnostics, based on observations and analysis of solar oscillations and waves, give insights into the physical processes in the solar interior and mechanisms of solar magnetic activity. In addition, simultaneous highresolution multi-wavelength observations of the solar corona provide opportunity to investigate in unprecedented detail the coronal dynamics and links to the interior processes. These capabilities are illustrated by initial results on the large-scale dynamics of the Sun, the subsurface structure and dynamics of a sunspot and observations of a X-class solar flare.
\end{abstract}

Keywords. Plasmas, Sun: helioseismology, magnetic fields, sunspots, photosphere, flares

\section{Introduction}

It is well accepted that the Sun represents a unique laboratory for plasma astrophysics. The most fundamental plasma processes in the Universe, including generation of magnetic fields by dynamo, magnetic self-organization in turbulent plasma, magnetic energy release, particle acceleration, MHD waves and shocks, are observed on the Sun in significantly more detail than in other astrophysical objects. Thus, investigation of these processes on the Sun can shed light in other cosmic astrophysics phenomena.

\section{SDO science goals and instrumentation}

The primary goal of the SDO mission is to study the origin of solar magnetism and variability, to characterize and understand the Sun's interior and how the physical processes inside the Sun are related to surface and coronal magnetic fields and activity. It carries three instruments, which observe the Sun uninterruptedly (except short eclipse periods in March and September) with high spatial and temporal resolution.

The basic characteristics of the instruments are the following:

- Helioseismic and Magnetic Imager (HMI) provides spectro-polarimetric observations in photospheric Fe 6173A line (Schou et al., 2010):

○ 4096x4096 images every $45 \mathrm{sec}$; spatial resolution $\sim 0.5 \mathrm{arcsec} /$ pixel $(\sim 350 \mathrm{~km})$;

- Doppler velocity;

$\circ$ Line-of-sight magnetograms;

- Vector magnetograms and plasma parameters every 12 minutes.

- Atmospheric Imaging Assembly (AIA) has 4 telescopes to obtain EUV images of the solar atmosphere and corona in 8 channels (Golub, 2006): 

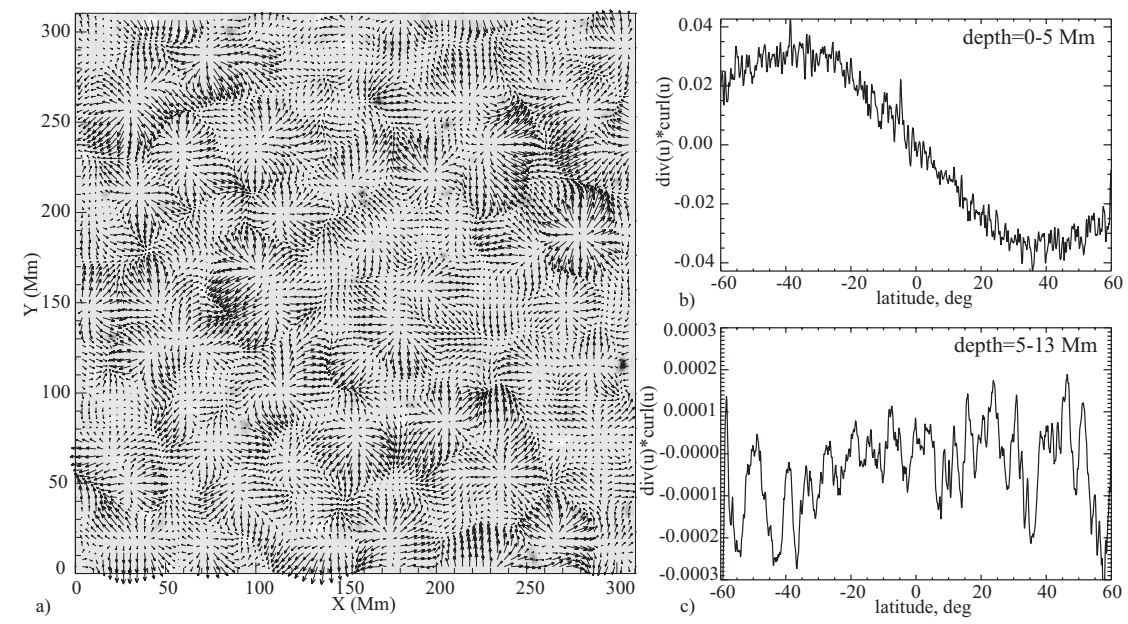

Figure 1. a) Subsurface flow field beneath a quiet-Sun region in the depth range of $1-3 \mathrm{Mm}$, obtained from the SDO/HMI data using the time-distance helioseismology pipeline (Zhao et al., $2011)$; the proxy of kinetic helicity, $(\boldsymbol{\nabla} \cdot \mathbf{u})(\boldsymbol{\nabla} \times \mathbf{u})_{z}$ as a function of latitude in two subsurface layers: b) 0-5 Mm and c) 5-13 Mm deep. The grayscale background in panel a) is the corresponding photospheric magnetogram showing concentrations of small-scale magnetic structures at the boundaries of supergranular convective cells.

○ 4096x4096 images every $3 \mathrm{sec}$; spatial resolution $\sim 0.5 \mathrm{arcsec} / \mathrm{pixel}$;

○ 7 EUV channels in a sequence of iron lines and He II $304 \AA$;

。 One UV Channel with $1600 \AA, 1700 \AA$, white light filters;

- Plasma temperature and differential emission measure of the solar transition region and corona are determined in the range of $10^{5}-10^{7} \mathrm{~K}$.

- EUV Variability Experiment (EVE) makes high-spectral resolution observations of UV irradiance using 2 main systems with 6 spectral channels (Woods et al., 2010):

- Multiple EUV Grating Spectrometer: 0.1-37 nm, $10 \mathrm{sec}$ cadence;

- EUV Spectrophotometer: 0.1-38 nm, 0.25 sec cadence.

The HMI and AIA collect $\sim 4$ TB of data per day. The data products are available from the Joint Science Operations Center at Stanford (http://jsoc.stanford.edu).

\section{Investigation of solar dynamo}

Investigation of the solar dynamo is one of the primary objectives of the SDO mission. The current paradigm is that the poloidal magnetic of the Sun is generated by turbulent helicity (so-called $\alpha$-effect) in the convection zone, primarily at the boundaries, in a subsurface shear layer and in the tachocline (a thin layer at the bottom of the convection zone), and that the toroidal magnetic field is generated in the tachocline by the differential rotation ( $\Omega$-effect). It is also assumed that the toroidal magnetic emerged at the surface at low latitudes in the form of bipolar magnetic active regions is transported by the meridional circulation to the polar regions, where it reverses the polarity of the dipolar poloidal field. Therefore, it is critical to obtain the information about the subsurface convection flows, variations of the differential rotation and meridional circulation. Such measurements are obtained through a helioseismology data analysis pipeline, which includes measurements of frequencies of normal oscillation modes of the Sun and inferences of the 2D sound-speed and rotation (radial and latitudinal) profiles (so-called global helioseismology), and also 3D maps of subsurface sound-speed perturbations and detailed 


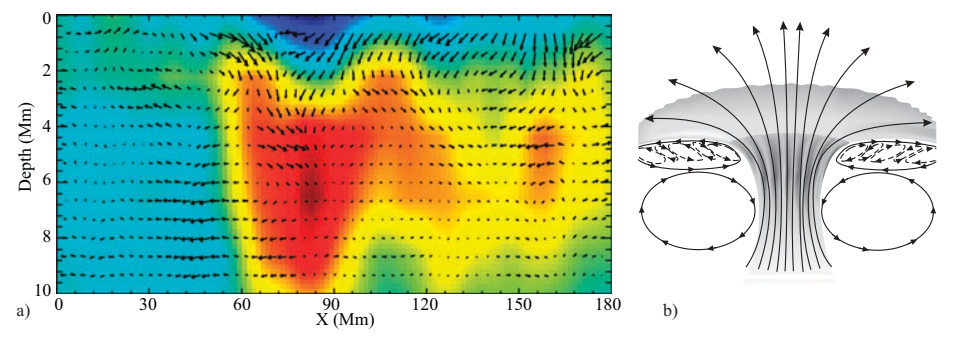

Figure 2. a) A vertical cut through a sunspot structure, obtained by inversion of acoustic travel-time variations from the SDO/HMI data analysis pipeline. The background shows variations of the wave speed, which are negative in a shallow subsurface layer and positive in the deeper interior. Arrows show plasma velocity with a typical speed $0.5-1 \mathrm{~km} / \mathrm{s}$, forming a converging flow pattern. b) a schematic illustration of a possible structure of subsurface flows: a thin layer of near-surface shear (Evershed) flows formed by magnetoconvection in inclined magnetic field, and deeper converging downflow, which maintains the compact magnetic structure.

flow in the upper convection zone, about $30 \mathrm{Mm}$ deep (local helioseismology) (see e.g. Kosovichev \& HMI Science Team, 2007; Zhao et al., 2011). Figure 1 shows a sample of the subsurface flow field, and estimates of a proxy of the kinetic helicity, $(\boldsymbol{\nabla} \cdot \mathbf{u})(\boldsymbol{\nabla} \times \mathbf{u})_{z}$, in two subsurface layers. It seems that the latitudinal dependence of the helicity parameter changes with depth. Initial results have been also obtained for the meridional circulation (Zhao et al., 2011). For investigations of the solar cycle it is important to monitor the large-scale solar dynamics over a significant portion of the solar cycle, and the mission plan is to observe at least the raising phase of the solar cycle and the maximum.

\section{Magnetic self-organization in solar plasma}

The solar magnetic field after emergence on the surface becomes quickly organized in stable structures, sunspots, which are stable for much longer than the convective turnover time. The local helioseismology and magnetic field observations with SDO/HMI provide unique data for studying the processes of formation and stability of the magnetic structures. Figure 2a illustrates initial results of local helioseismology imaging of the subsurface structure and plasma flows beneath a stable sunspot. These results confirm the previous evidence from analysis of the SOHO/MDI and Hinode data (Zhao et al., 2010) that the magnetic self-organized structures are formed and maintained by converging flows and downdrafts in subsurface layers as illustrated in Fig. 2b, and supported by recent numerical simulations (Kitiashvili et al., 2010).

\section{Solar flares and magnetic energy release}

The understanding of magnetic energy release in solar flares is one of the outstanding problems of plasma astrophysics. The AIA instrument provides high-resolution highcadence images and diagnostics of temperature and differential emission measure of plasma in the solar corona. The HMI measures detailed configuration of the photospheric magnetic fields and their variations in flaring regions. The EVE monitors the UV flux and has made an important discovery of a secondary peak of the EUV emission in solar flares, which has about four times more energy than the EUV energy during the time of the X-ray flare peak. Together with the high-resolution Hinode data and X- and $\gamma$-ray measurements from the RHESSI and FERMI missions we have now a unique collection of data for understanding the mechanism of solar flares and their effects in the solar interior and atmosphere. Figure 3 illustrates the photospheric and helioseismic effects of the first X-class flare, observed by SDO (Kosovichev, 2011). The flare occurred in a 

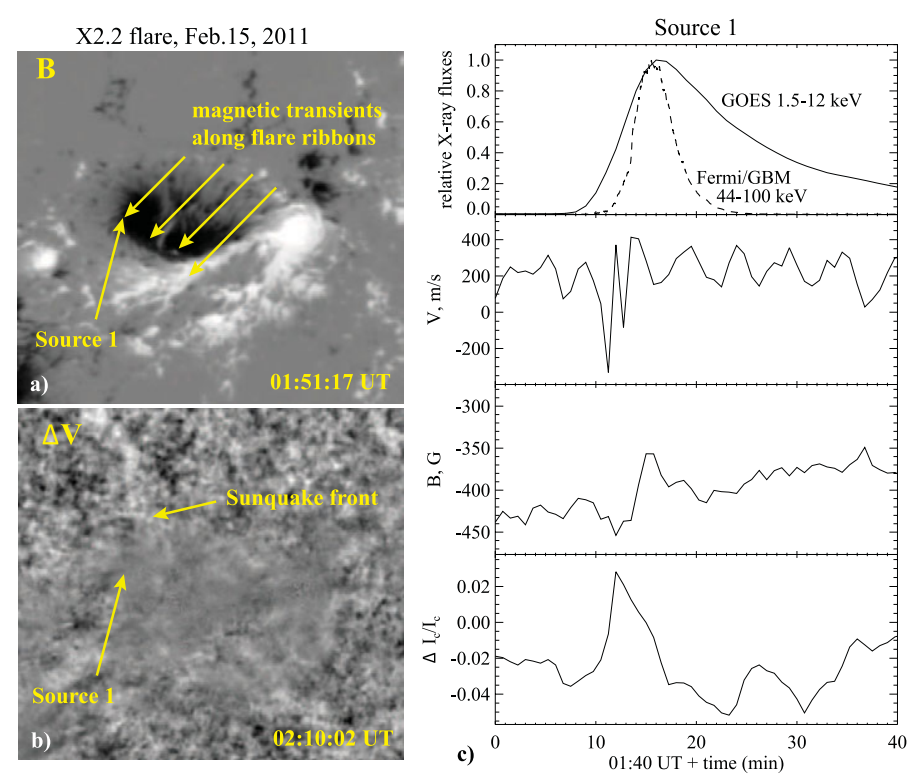

Figure 3. SDO/HMI observations of the impact of the X2.2 solar flare (15 Feb 2011) on the photospheric plasma: a) magnetogram showing the magnetic transients on along both sides of the magnetic neutral line during the impulsive phase; b) the Dopplergram difference taken about 20 minutes after the impact showing a circular helioseismic wave ('sunquake') originating from the impact place (Source 1); c) variations of the soft and hard X-ray emissions measured by GOES-15 and Fermi/GBM instruments, and variations of velocity, magnetic field and continuum intensity in Source 1 from HMI (Kosovichev, 2011).

$\delta$-type sunspot near the magnetic neutral line. The HMI magnetograms, Dopplergrams, and continuum intensity images reveal strong impacts in the photosphere during the impulsive phase. The flare impacts, which are believed to be caused by precipitation of high-energy particles, are organized in two ribbons rapidly expanding from the neutral line (Fig. 3a). In one place (marked 'Source 1'), the flare impact initiated a strong helioseismic wave ('sunquake'), observed as expanding circular-shaped ripples on the surface (Fig. 3b). Comparison of the photospheric impact with the flare X-ray emission observed with the GOES-15 and FERMI/GBM instruments shows that the photospheric impact correlates with the initial soft X-ray increase, and not with the following hard X-ray impulse as thought before. These observations challenge the standard model of solar flares.

\section{Acknowledgements}

I thank the participants of the ISSI team on solar magnetism for stimulating discussions, and the ISSI (Bern) for support.

\section{References}

Golub, L. 2006, Sp. Sci. Rev., 124, 23

Kitiashvili, I. N., Kosovichev, A. G., Wray, A. A., \& Mansour, N. N. 2010, ApJ, 719, 307.

Kosovichev, A. G., \& HMI Science Team 2007, Astron. Nach., 328, 339

Kosovichev, A. G. 2011, arXiv:1102.3954

Schou, J., et al. 2010, Sol. Phys, in press.

Woods, T. N., et al. 2010, Sol. Phys, in press

Zhao, J., Kosovichev, A. G., \& Sekii, T. 2010, ApJ, 708, 304

Zhao, J., et al. 2011, Sol, Phys., submitted. 